¿Institucionalización o desinstitucionalización del curso

de vida? Biografía y sociedad como un enfoque

integrativo e interdisciplinario*

\title{
Ludger Pries
}

El objetivo de este trabajo es presentar brevemente la manera como se ha utilizado y de sarrollado el enfoque "biografia y sociedad" en Alemania. Este enfoque constituye una visión de la realidad social que enfatiza la dominación del tiempo y la relación del individuo con la sociedad. De manera especial, se hace referencia a la hipótesis de la institucionalización del curso de vida y el papel que algunos factores han jugado en ésta, como por ejemplo, el aumento de la esperanza de vida.

Para ponderar la fuerza explicativa de este enfoque, se analizan algunos datos ob tenidos para el caso en la ciudad de Puebla. Finalmente, lo anterior permite reflexionar. sobre las posibles lineas de investigación futuras respecto a este tema.

\section{Introducción}

El concepto de "ciclo de vida" o de "curso de vida" es básico no solamente para la demografía, sino también para las ciencias sociales. E1 profesional de esta ciencia no puede analizar el comportamiento demográfico sin conocer, por ejemplo, las pautas temporales de fecundidad en las trayectorias de vida de los individuos; así como el sicólogo desarrolla una idea de la secuencia de las capacidades cognoscitivas durante la niñez y la:juventud de los hombres y de los problemas psicosociales primordiales en cada etapa de su vida, para el sociólogo es de gran interés, por ejemplo, la manera en que las diferentes fases socialmente definidas de la vida (niñez, juventud/educación/formación, vida de adulto, vejez) están cambiando su ubicación y extensión temporal y su peso relativo en las sociedades.

En este contexto, presentamos aquí una tradición y un campo de investigación específicos, surgidos en las últimas dos décadas -principalmente en Alemania-, que podemos denominar como enfoque biografia y sociedad. Tal corriente se desarrolló sobre todo en la investigación sociológica, pero es una visión interdisciplinaria e integral: no se trata de un nuevo paradigma cerrado, ni consiste tan sólo en una nueva me-

* Agradezco a Orlandina de Oliveira y a algunos dictaminadores anónimos los valiosos comentarios que hicieron a la primera versión de este trabajo; por supuesto, todos los errores son responsabilidad mía. 
todología. Lo que llamamos enfoque biografía y sociedad es más bien una percepción específica de la realidad social, que enfatiza primordialmente la dimensión del tiempo y la relación del individuo con la sociedad. Según lo en tendemos aquí, biografia y sociedad considera una perspectiva hacia las trayectorias de vida -en el sentido de las "secuencias objetivas y claramente medibles de los individuos, según su posición social"-, así como una visión de las historias de vida, es decir, de las construcciones subjetivas que desarrollan los hombres sobre el pasado, presente y futuro de su propia vida dentro del contexto social en el que están inmersos.

En las ciencias sociales éste puede fungir como un concepto integrativo y fructífero; representa un enfoque especial respecto a la manera como las vidas de los hombres y la sociedad se estructuran mutuamente; considera el hecho de que toda realidad social tiene su tiempo y, por lo tanto, para entenderla hay que complementar cortes trasversales y longitudinales - perspectivas ambas que siempre estuvieron más presentes en la demografía que en otras disciplinas-; por último, permite la integración tanto de las corrientes metodológicas "positivistas" o cuantitativas, como de aquellas de corte interpretativo o cualitativo.

Después de esbozar brevemente los antecendentes generales del enfoque biografía y sociedad, en la primera parte de este trabajo se resume el desarrollo del mismo, para el caso de Alemania; en la segunda parte se revisan algunas hipótesis relativas al tema y, con base en ellas, se intenta mostrar en la tercera parte la "fuerza explicativa" de este concepto al analizar algunos datos de una investigación empírica realizada en la ciudad de Puebla, que finalmente nos hará reflexionar sobre las posibles líneas futuras de investigación.

\section{Antecedentes, fuentes y delimitantes del enfoque biografia y sociedad}

En cierta forma, el enfoque biografía y sociedad corrió en lo general la misma suerte que los grandes paradigmas de las ciencias sociales: después de una primera etapa en la que se sentaron sus fundamentos, y aun con una visión bastante amplia e integradora, empezó un periodo de "maduración" y de diferenciación interna, que frecuentemente resultó en la delimitación mutua de "trincheras" de "subescuelas", en las cuales destacaron más los matices diferenciadores que las ideas comunes; en una tercera fase se vuelven más pragmáti- 
cas las "subescuelas" ya establecidas y se presenta una etapa donde surge un nuevo acercamiento. ${ }^{1}$

En términos generales, en el caso del enfoque biografía y sociedad la primera fase del auge se inicia con los grandes estudios de lo que después se llamaría Escuela de Chicago y termina más o menos con el fin de la segunda guerra mundial (el famoso libro Street Corner. Society de William Foote Whyte, por ejemplo, se publicó en 1943). Una visión aun holística de biografía y sociedad se presenta en W. I. Thomas y F. Znaniecki (1921), en las investigaciones sobre los migrantes polacos en Europa y Estados Unidos; estos autores afirman (p. 1831):

The human personality is both a continually producing factor and a continually produced result of social evolution, and this double relation expresses itself in every elementary social fact; there can be for social science no change of social reality which is not the common effect of pre-existing social values and individual attitudes acting upon them, no change of individual consciousness which is not the common effect of pre-existing individual attitudes and social values acting upon them.

En estos estudios realizados en la Universidad de Chicago, se reconstruyó la vida personal de los campesinos polacos que migraron a Estados Unidos, la "desorganización de la familia", la "desorganización de la comunidad”, etc. Siguieron otras investigaciones donde, por ejemplo, las vidas individuales fueron reconstruidas por sus fases muy particulares de niñez y:juventud, de formación y estudios, la fase de actividad económica y la del envejecimiento, donde las variaciones socioculturales de una sociedad se investigan por medio de la observación de los cambios ocurridos entre distintas generaciones. Como denominador común de esta visión se puede destacar la idea de que no existen lo social y la sociedad fuera de los hombres y de que no existen hombres fuera de la sociedad y de lo social. De esta forma fueron superadas las yuxtaposiciones tan frecuentes en las ciencias sociales $-y$ no por ello menos infructuosas- de "individuo y sociedad", "actor y estructura" y "objetividad y subjetividad".

${ }^{1}$ Estamos conscientes de que esto es una simplificación exhaustiva: habría contradicciones y discusiones en la primera etapa, probablemente existieron intentos integradores en la segunda, y la tercera fase seguramente no se caracteriza por una situación de armonía sin "trincheras"; respecto de la existencia de este tipo de "coyunturas paradigmáticas" generales, véanse por ejemplo, Alexander et al., 1987; Beck, 1986; Giddens et al., 1990 y Long y Long, 1992. 
Mientras que los estudios de la Escuela de Chicago en cierta forma se pueden considerar como pioneros, integradores y de una alta riqueza empírica y teórica, en las tres décadas de la posguerra -y no sólo en Estados Unidos- el enfoque biografía y sociedad perdió terreno e importancia. ${ }^{2}$ Fue en la segunda mitad de los años setenta cuando este campo recuperó su importancia y apenas en los años ochenta logró un renacimiento notable. En Estados Unidos, Alemania y Francia se realizaron entonces investigaciones sobre "ciclos de vida" y sobre "trayectorias de vida" utilizando una metodología predominantemente cuantitativa con un sesgo hacia el racionalismo crítico; al mismo tiempo, florecen la "investigación biográfica" y los estudios de "historia de vida" como la corriente más cualitativo-interpretativa del enfoque biografía y sociedad, la cual registra avances muy importantes. ${ }^{3}$

Este auge en el campo de biografía y sociedad va acompañado con la aplicación de métodos cuantitativos y cualitativos cada vez más complejos. Respecto a los primeros, el event analysis gana una importancia cada vez mayor. Contrastando, por ejemplo, con las tablas de movilidad aplicadas frecuentemente en la investigación sobre movilidad y estructura sociales (Herz, 1986), el enfoque de ciclos, carreras y trayectorias de vida analiza la secuencia consecutiva y completa de individuos (u otras unidades de análisis) por posiciones e intenta explicar "eventos ocurridos" según "eventos anteriores"; con esto, el eje de tiempo gana un peso propio como variable explicativa. La lógica del "análisis de eventos" implica un "corte longitudinal" completo, no solamente "cortes transversales" en dos puntos de tiempo (por ejemplo, antes y después de cierto acontecimiento) o en más puntos de tiempo (lo común en investigaciones de tipo panel). ${ }^{4}$

Respecto a los métodos cualitativos, la investigación sobre "historias de vida" y "relatos de vida" se deriva de las tradiciones de las ciencias sociales interpretativas o reconstructivas, como, por ejemplo, el interaccionismo simbólico y la etnometodología, la hermenéutica, la sociolingüística y la sicología social. Anteriormente, el procesamiento

\footnotetext{
${ }^{2}$ Respecto a este balance, véanse por ejemplo, Balán et al., 1974; Eider y Caspi, 1990; Pujadas, 1992 y Saltamalacchia, 1992.

${ }^{3}$ Para el caso de Alemania, en el cual nos concentramos aquí, véanse para lo primero, Hagestad, 1990 y Mayer, 1990; y para lo segundo, Fuchs, 1984 y Kohli y Robert 1984.

${ }^{4}$ Véanse, por ejemplo, para Estados Unidos, Noruega y Alemania las investigaciones citadas en Allmendinger, 1989: 72; Bertaux y Kohli, 1984; Eider y Caspi, 1990, y Mayer, 1990.
} 
de datos cualitativos (como los textos hablados, el lenguaje corporal o los símbolos y emanaciones culturales) tenía cierta aura de arbitrariedad y, a veces, se obtenía un resultado como si de una "caja negra" saliera una interpretación exhaustiva, sin hacer explícito el procedimiento metodológico.

En cambio, el nuevo auge de la investigación sobre "historias de vida" va acompañado del avance de métodos muy elaborados y ambiciosos (como es el caso de la "hermenéutica objetiva" de Oevermann), e incluso de software para preparar el análisis de datos verbales. ${ }^{5} \mathrm{Al}$ mismo tiempo, en los años ochenta, en países como Estados Unidos, Alemania o Francia observamos cierto acercamiento entre las dos líneas de investigación de "trayectorias de vida" y de "historias de vida" (véanse por ejemplo, Voges, 1987 y Dex, 1991). Esto se debe en parte a la tendencia generalizada de "detención" entre paradigmas diferentes, que antes se encontraban más directamente en disputa.

Aunque México no está totalmente al margen de estas grandes tendencias, coyunturas y corrientes teórico-paradigmáticas, sí tiene su propia dinámica particular. Cuenta con una muy rica tradición de estudios de tipo biografía y sociedad y aparentemente el "cisma" entre el enfoque de "trayectorias de vida" y el de "relatos de vida" no fue tan fuerte como en otros países. Fjemplo de esto son tres importantes estudios practicados sobre las ciudades más grandes del país: Monterrey, México y Guadalajara. A mediados de los años sesenta Jorge Balán, Harley Browning y Elizabeth Jelin (1977) realizaron un estudio pionero en la ciudad de Monterrey. Influidos por las tradiciones de la Escuela de Chicago averiguaron los procesos de migración del campo y de inserción a la vida y al trabajo urbanos. Algunos años después, Humberto Muñoz, Orlandina de Oliveira y Claudio Stern (1977), con preocupaciones parecidas, llevaron a cabo una amplia investigación en la ciudad de México. Diez años después, al principio de los ochenta, Agustín Escobar, Mercedes González de la Rocha y otros levantaron encuestas de trayectorias de vida y realizaron entrevistas de historias de vida en Guadalajara, la tercera ciudad más grande de la República (Escobar, 1986; González de la Rocha, 1986). Estos tres estudios se caracterizan por su enfoque holístico-integral y por la combinación de métodos cuantitativos y cualitativos, por lo que se consideran obras de gran valor.

${ }^{5}$ Véanse para Europa, Bertaux y Kohli, 1984, y para Alemania, Schütze, 1983; Fuchs, 1984; Fischer y Kohli, 1987, y Bohnsack, 1991. 
Además de este núcleo de investigaciones integrales, en México se cuenta con una larga tradición tan to de estudios de historias o relatos de vida, como de trayectorias de vida. Algunos estudios sobre la tradición de historia de vida aplicados, por ejemplo, sobre historias de familias, realizan una descripción y un seguimiento concreto de la trayectoria y las percepciones de vida de individuos o de grupos familiares, de los factores influyentes, constituyentes y explicativos del curso de estas vidas, etc. Sus preguntas centrales tratan de aclarar dos cuestiones: ¿en qué contexto social se puede entender o cuáles factores pueden explicar la trayectoria concreta de estas vidas singulares o familiares? Metodológicamente recurren al análisis de materiales personales como cartas, diarios, etc., al examen de documen tos coetáneos, y a entrevistas con las personas involucradas o con expertos. Un ejemplo mundialmente famoso es el libro Los hijos de Sánchez de Oscar Lewis (1965); también hay obras importantes sobre familias de políticos (Camp, 1990) o empresarios (para la región de Puebla, véanse por ejemplo, Gamboa, 1985 y Torres, 1994); trabajos más recientes en esta línea cualitativa son, por ejemplo, Mummert, 1990; Wilson, 1990 y García y Oliveira, 1994.

También hay, sobre todo desde la perspectiva de la demografía, aportaciones importantes a la tradición de las trayectorias que emplean métodos cuantitativos avanzados (véase Bronfman, 1990, así como los aportes al número 1 de la Revista Mexicana de Sociología, 1990) y a la revista Estudios Demográficos y Urbanos, núms. 2 y 3, en especial el de Suárez López (1992). De cierta manera, los estudios que enfocan la situación de la mujer, sobre todo el tema "mujer y trabajo", y la unidad doméstica como unidad de análisis, representan un campo temático donde las vertientes cuantitativas y cualitativas, de "trayectoria de vida" y de "historia de vida" se encuentran e intercambian, y donde podemos notar innovaciones notables (véanse, entre otros, Cooper et al., 1989; Oliveira et al., 1989a y Oliveira, 1989b).

Sin contar con un estudio amplio y profundo de la historia de las investigaciones sobre trayectorias e historias de vida en México, un rastreo de la bibliografía da la impresión de que México es uno de los países que ofrecen mayor interés y más riqueza en el tema de biografia y sociedad. En su tiempo los estudios realizados estaban muy actualizados en lo relativo a los métodos aplicados (path analysis, en el estudio de Monterrey, o análisis de regresiones en los de la ciudad de México, por ejemplo). Pero, en términos globales, parece que a partir de los años ochenta el enfoque biografía y sociedad 
fue perdiendo su dinámica y su fuerza aglutinadora. No ocurrió lo mismo en la mayor parte de los países europeos, particularmente en Alemania.

\section{¿Institucionalización del curso de vida en Alemania?}

A partir de los años setenta, al igual que ocurría en otros países, en Alemania las investigaciones sobre trayectorias de vida (Lebensverlaufsforschung) y sobre historias de vida (Biographieforschung) tenían un auge considerable. En una primera fase estas dos "subescuelas" se desarrollaron independientemente $y$, a veces, con muchas controversias entre lo que se puede llamar la "línea dura" de trayectorias de vida y la "línea blanda" de historias de vida. En un segundo periodo, a partir de la mitad de la década de los ochenta, estas dos corrientes se acercaron cada vez más y, de cierta forma, "salieron de sus trincheras".

Aunque no se terminaron las polémicas entre los científicos más orientados hacia métodos cuantitativos y aquellos más afines a los métodos cualitativos, se logró un entendimiento y una base científica común al afirmar y destacar la importancia, primero, del "corte longitudinal" o la historización de las vidas y de la realidad sociales y, segundo, del trato dialéctico de los conceptos individuo y sociedad como dos perspectivas analíticas diferentes sobre el mismo objeto de la vida y de la convivencia social de los hombres. Con sus diversas ramificaciones, este enfoque biografía y sociedad ganó mucha fuerza en las ciencias sociales en general y particularmente en la sociología; por ejemplo, en los años ochenta se crearon las secciones correspondientes en las asociaciones profesionales y científicas, aparecieron varias compilaciones muy valiosas sobre la materia y las revistas sociológicas más importantes dedicaron números especiales a este tema. ${ }^{6}$

Resumiendo los avances logrados en los diferentes aspectos, Kohli y Robert (1984) definen la biografia en un sentido amplio que abarca, por un lado, la (re)construcción subjetiva de las vidas y por otro, la realidad social de movimientos de personas en posiciones y situaciones sociales. El enfoque biografia y sociedad, por lo tanto, integra la investigación de las pautas y regularidades individuales de las trayectorias de

\footnotetext{
${ }^{6}$ Respecto a las compilaciones y monografías, véanse por ejemplo Fuchs, 1984; Kohli y Robert, 1984; Voges, 1987; ejemplos de ediciones especiales de revistas son Berger y Hradil, 1990, y Mayer, 1990.
} 
vida -en el sentido de secuencias de unidades sociales (in dividuos, unidades domésticas, organizaciones, etc.) en posiciones sociales (niñez, adolescencia, formación escolar y profesional, entrada y movilidad en el empleo, matrimonio, paternidad, jubilación, etc.) - con la tradición de estudios sobre historias de vida, entendidas como conceptos e interpretaciones institucionalizadas de la vida y como el significado y la (re)construcción de la ubicación y la relación de los hombres en su ambiente social abarcando su pasado, presente y futuro.

Tomando en cuenta estas dos perspectivas analíticas diferentes, el enfoque biografía y sociedad se concentra en explicar y enten der la estructuración de la sociedad por las trayectorias y las historias de vida individuales y colectivas y, en sentido opuesto, estudiar la estructuración de las trayectorias y las historias de vida individuales y colectivas por las instituciones y procesos sociales. En este sentido, no es el objetivo de este enfoque ver, por ejemplo, cómo los "sujetos individuales" actúan -más o menos libremente- en las "estructuras objetivas sociales". Más bien, como lo expresó Norbert Elias, no hay sociedad fuera de los hombres y no hay hombres fuera de la sociedad. Resulta que no es el individuo el tema de la investigación biográfica, sino el producto social de la biografía como muestra o pauta de trayectoria y de orientación en el mundo social cotidiano e histórico.

Para el hombre singular, el mundo en que se halla, en el cual se orienta y actúa, ya está hecho. El individuo está confrontado con una realidad estructurada y ordenada por símbolos y significados, por instituciones y organizaciones, por relaciones de poder y de emoción, por normas de comunicación y de comportamiento. En este sentido, el hombre -es decir, el individuo- ni puede ni tiene que inventar las pautas para pensar o actuar, sino que las encuentra ya listas y producidas en el mundo social.

Sin embargo, según el enfoque biografía y sociedad, esto solamente refleja la mitad de la verdad o de la realidad: para el hombre singular el mundo no está cerrado, la realidad cotidiana y las "reglas del juego prefabricadas" siempre contienen "huecos negros" y espacios por llenar. No hay una "realidad objetiva cerrada", sino que el individuo está autorizado y se ve obligado a interpretar situaciones y reglas, a reducir las informaciones y realidades accesibles, a votar por opciones y a construir nuevas reglas y realidades. Resulta que no se trata de una "realidad objetiva homogénea", la cual deja determinados espacios de adaptación y de orientación al sujeto, sino, más bien, se trata de un proceso complejo de 
génesis, interpretación, aplicación, adaptación y de cambio de las "reglas del juego" o del modus operandi: "Entendemos 'la biografia' como constructo del mundo cotidiano, lo cual contiene la ambigüedad del mundo de la vida como una regularidad prefijada y, al mismo tiempo, como una realidad emergente" (Fischer y Kohli, 1987: 35).$^{7}$

\section{La hipótesis de la institucionalización del curso de vida}

Es obvio que el enfoque biografia y sociedad es mucho más que un puro método o una metodología adicional; más bien integra un marco teórico-paradigmático -sobre cómo conceptuar "individuo y sociedad", "estructura y acción", "objetividad y subjetividad"- con una visión metodológica de "hacer cortes longitudinales" para entender y explicar la realidad social. Un buen ejemplo de la envergadura del enfoque biografía y sociedad es la hipótesis de la "institucionalización del curso de la vida" en sociedades contemporáneas, desarrollado para Alemania principalmente por el sociólogo Martin Kohli (1985).

Esta hipótesis parte de la idea de que, para investigar el cambio social, no basta medir, por ejemplo, entre otros elementos, la transmutación de la estructura de actividades y posiciones económicas, los cambios en la composición numérica de la familia o el comportamiento generativo en la sociedad. Más bien, las pautas de las biografías mismas son un indicador o un reflejo central donde se plasman tanto las regularidades, rutinas o "estructuras" sociales, como el cambio social. Las pautas intersubjetivas y el manejo individual del tiempo representan dimensiones muy importantes del análisis de las sociedades, de tal forma que las mismas pueden ser estructuradas y caracterizables por modelos diferentes de biografías, considerando los dos sentidos: de trayectorias y de historias de vida.

Según Martin Kohli, un aspecto central del cambio de sociedades como la alemana es la institucionalización del curso de vida. En la actualidad la biografía es una institución social en el sentido de un sistema de reglas de acción y de expectativas compartidas. Eso no necesariamen-

\footnotetext{
${ }^{7}$ En lo concerniente a la ambigüedad entre estructura y acción respecto a la biografia, véanse también Brose, 1990 y Fuchs-Heinritz, 1990. Como pioneros en desarrollar los fundamentos de esta perspectiva integral hay que mencionar a Alfred Schütz y algunos de sus discípulos como Peter Berger y Thomas Luckmann. El término modus operandi es central en el enfoque de la "hermenéutica objetiva" de U. Oevermann et al., 1979.
} 
te es ni siempre fue así. Durante el largo proceso de modernización desarrollado en los pasados cuatro siglos -entendiendo el término no como concepto normativo sino en el sentido de una transformación histórico-empírica (Pries, 1993) - se cambiaron paulatinamente la pauta y el régimen de las trayectorias e historias de vida. A muy grandes rasgos, esta transformación de la biografia se caracteriza por el traspaso de una pauta de la vida como una aglomeración de casualidades y de suerte hacia una pauta de la vida como una trayectoria previsible, contable y planeable. De esta forma, la biografia se institucionaliza como un conjunto de regularidades observables y de reglas y prácticas de expectativas, aspiraciones, conceptos y acciones. Según Martin Kohli podemos concretar este cambio en cuatro aspectos:

a) Duración de la vida y papel de la muerte

En la Edad Media y aun en el siglo pasado la muerte podía ocurrir casi en cualquier momento del transcurso de la vida debido a las guerras de tribus, enfermedades y epidemias, catástrofes naturales, etc. La muerte no fue considerada como un "evento de la vida" que con regularidad ocurre o "se acerca" a cierto punto en la trayectoria, por ejemplo a la edad de 50 o 60 años; más bien, la probabilidad de morir fue casi la misma en los diferentes periodos de la vida de una persona. En los últimos cien años la duración promedio de la vida aumentó mucho, aunque su duración máxima casi permaneció igual. En Alemania, según argumenta Kohli (1985), entre 1871 y 1881 , de las mujeres que cumplieron 20 años $17.2 \%$ murió antes de cumplir 40 años, y entre las que cumplieron esa edad, $29.6 \%$ murió antes de cumplir 60 años. En cambio, en el periodo 1979-1981 los valores correspondientes son $1.5 \%$ y $7.3 \%$, respectivamente. Entre 1881 y 1890 , de todos los hombres, solamente $19.7 \%$ cumplió 70 años, mientras que entre 1979 y 1981, 72.2\% llegó a la edad límite para la jubilación, es decir, 65 años.

Este proceso de la concentración de la muerte en periodos de edades mayores se dio en casi todos los países en proceso de modernización sociohistórica. La gráfica 1 muestra las curvas de sobrevivencia acumulativas según cohortes para Estados Unidos, de 1840 hasta 1980. De todas las personas que murieron en 1840, los bebés que tenían un año muestran una alta probabilidad de no sobrevivir el próximo "evento", en este caso, el siguiente año calendario. Pero después de esta fase de mortalidad infantil elevada, los muertos del siglo pasado 
se repartieron de forma casi homogénea sobre toda la trayectoria de edades. En cambio, analizando la curva de mortalidad del año 1980 -dada la pendiente muy acentuada de la línea correspondiente entre los 60 y 90 años-, se revela una fuerte concentración de la muerte a estas edades. Afirma Kohli (1985: 5): "con respecto a la prolongación de la duración de la vida no es decisivo el aumento de la media, sino el decrecimiento de la varianza".

En general podemos constatar que la muerte, partiendo desde una casi omnipresencia en todas las etapas de la vida, está cada vez más "marginalizada" en una fase de edad muy específica. Con esto el transcurso de la vida pierde su arbitrariedad respecto del "evento" de la muerte y se establece cierta probabilidad de que este "evento" ocurra con alguna regularidad a cierta edad. De esta manera la trayectoria de vida gana cierto perfil de secuencia de diferentes etapas, regla que a su vez estructura las expectativas y los planes de los hombres.

\section{GRÁFICA 1}

\section{Curvas de sobrevivencia según cohortes en Estados Unidos}

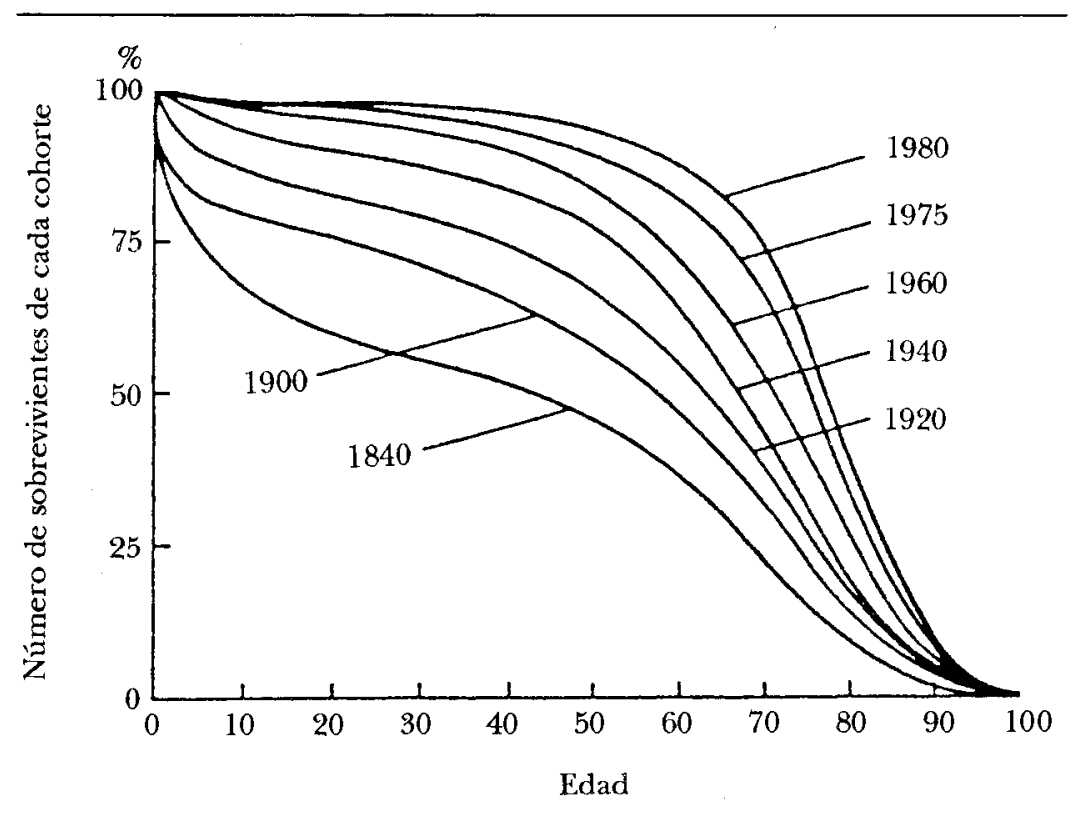

Fuente: Kohli (1985: 5). 
b) Cambios en la familia

Antes de la "institucionalización del curso de vida", en Alemania, como en muchos países, no existía un ciclo propio y muy definido para el desarrollo de la familia en el sentido de una clara concentración del matrimonio o nacimiento de los niños en determinada edad. Más bien el casarse, unirse libremente sin casarse, o tener niños, no fueron "eventos" claramente concentrados o limitados a determinado periodo de la biografia. Habíajóvenes que ya tenían hijos desde los 16 años y también personas ya viejas se unían o tenían hijos. Respecto a estos "eventos", el proceso de la "institucionalización del curso de vida" se refleja en el hecho de que se está perfilando cada vez más un periodo propio de pareja sin hijos (empty nest), se da una concentración de nacimientos de hijos en un periodo no muy lejano al casamiento, existe un lapso más corto entre los nacimientos de los hijos y se tiene un promedio menor de ellos.

Como ejemplo, Martin Kohli cita el estudio de Peter R. Uhlenberg sobre diferentes tipos de biografías de mujeres en Massachusetts. De los siete tipos de trayectorias, uno era caracterizado como el "tipo de familia normal" (en el sentido de la institucionalización del curso de vida): casamiento, tener niños, vivir:junto con la pareja hasta como mínimo los 55 años. Según el estudio, desde 1830 hasta 1920 el peso de este "tipo normal" se aumentó de $20.9 \%$ hasta $57.1 \%$. Lo que actualmente llamamos "tipo normal de familia" puede caracterizarse por algunos elementos centrales: la familia contiene dos (y no, como antes, tres o más) generaciones; esta "familia pequeña" tiene una importancia relativa mayor que la que anteriormente tenía la unidad doméstica más grande y más difusa; el tiempo del traspaso a una unidad doméstica u hogar propio (salida de la familia de origen, casamiento, establecimiento de un hogar independiente) es más corto, y esto tiene como consecuencia que la fisura entre la juventud y el mundo de los adultos sea más aguda; las diferencias y variaciones entre las edades de las parejas se reducen y normalizan.

Resulta que lo que después de la segunda guerra mundial se conoció como el "tipo normal de familia", en el siglo pasado constituyó nada más una de varias pautas de biografias. Otra vez el proceso de institucionalización del curso de vida se caracteriza por una reducción muy fuerte de las varianzas de tiempos y periodos y se establece, por un lado, por la emergencia de regularidades "objetivas" y observables y, por otro lado, por la significancia creciente que adquieren los conceptos y proyectos "normales" individuales y subjetivos que los actores tienen de su vida. 
c) Introducción de límites formales de edades

Un tercer elemento del largo proceso histórico de la institucionalización del curso de vida es la tendencia de los estados a introducir límites formales de edades para ciertos asuntos públicos. Un primer intento importante para introducir estos límites de edad en Europa fue, a principios del siglo pasado, el Código Napoleónico; éste consistió en una definición clara de límites de edades para, por ejemplo, asumir la responsabilidad penaljurídica, adquirir el derecho a votar, realizar el servicio militar y cumplir con la obligación de asistir a una escuela primaria. Con tales normas de edades mínimas o máximas para ciertos derechos u obligaciones la trayectoria de vida tomó cuerpo en el sentido de constituir una estructura universal de puntos o periodos en las vidas individuales.

Hoy día la institucionalización del curso de vida se refleja en la segmentación institucionalizada del sistema formal de educación o formación y de:jubilación. Podemos hablar de una división tripartita de la trayectoria de vida en niñez-formación, adulto-empleo y vejezjubilación con límites de edades definidas casi con precisión. En forma similar el empleo dependiente-asalariado se convirtió en la "relación normal de trabajo" y agudizó la separación entre el lugar de la familia o vivienda y el lugar de la actividad económica y, consecuentemente, marcó más claramente la separación de episodios distintos durante la trayectoria de vida.

d) Desarrollo de perspectivas biográficas propias

Un cuarto aspecto clave del proceso de modernización es el creciente "control de los afectos" (Elias, 1969) o el traspaso del control externo hacia el control interno (Weber, 1980; Riesman, 1958; Foucault, 1977). En relación con el concepto de vida esto significa, por ejemplo, una planeación explícita de la vida individual. Esta planeación y este "manejo de vida sistemático" (Max Weber) forman el eje central de la emergente identidad individual. Mientras que antes tenía mucho más peso una identidad colectiva como familia campesina grande, la identidad individual ganó terreno y se perfiló con la estructuración cada vez mayor y más contable de la vida que a su vez amplió el horizonte temporal de planificación de la vida.

Este cambio se refleja, por ejemplo, en las autobiografias de finales del siglo xviı: durante mucho tiempo las autobiografias -cuyo auge a su vez afirma el proceso de individualización y desarrollo de perspectivas biográficas propias- se caracterizaron por el intento de reconstruir fiel- 
mente las secuencias de eventos "objetivos" en un sentido cronológico. Al término del siglo xviI esta situación empezó a cambiar y las autobiografías mostraron cada vez más la preocupación del escritor por explicar y desenvolver sus conceptos y proyectos de vida individuales y psicosociales.

Resumiendo los elementos aquí esbozados de la hipótesis de la institucionalización del curso de vida, este planteamiento de Martin Kohli parece muy fructífero para entender y conceptuar el cambio social de una forma diferente. En este caso, el cambio social no es nada estéril o abstracto que sólo o primordialmente se perciba en el ámbito de las "estructuras" que existen fuera e independientemente de los hombres. Más bien se analiza como un cambio de pautas, de prácticas y de normas de trayectorias e historias de vida. En este sentido, el estudio estático de la llamada "estructura social" -como uno de los objetos centrales de todas las ciencias sociales y en especial de la sociología- se transforma en el análisis dinámico de la "estructuración social" (Giddens, 1984), que se realiza por instituciones sociales como la biografia.

Para los países altamente industrializados la hipótesis de la institucionalización del curso de vida tenía y tiene mucha fuerza explicativa $\mathrm{e}$ interpretativa, independientemente de que fue y es muy discutida. Algunos autores, como Ulrich Beck (1986), la retoman y diagnostican una creciente "desestandarización del ciclo familiar" y una flexibilización de la "trayectoria normal" y de las normas de edades. Según Beck, al final de este siglo vivimos un nuevo proceso de individualización y de vencimiento de uno de los últimos criterios de adscripción que-además del género- es la edad.

Sea cual fuere la respuesta concreta a la pregunta de si (aún) vivimos un proceso de institucionalización de la biografia o (ya) entramos a un periodo de creciente desinstitucionalización, queda suficientemente claro que el enfoque biografía y sociedad y la hipótesis de la institucionalización del curso de vida pueden abrir nuevos caminos del análisis y han comprobado su utilidad para entender las dinámicas de países como Alemania, Francia o Estados Unidos. Pero, ¿cuál es la situación en un país como México? ¿También se da un proceso de estandarización y de institucionalización del curso de vida?

¿Estandarización de trayectorias de vida en México?

Para responder a esta pregunta con precisión y fundamentos se necesitaría contar con datos cuantitativos y cualitativos adecuados para llevar 
a cabo un procesamiento de tipo "análisis de evento"; tal información es muy escasa en México. Las grandes encuestas y censos nacionales normalmente no ofrecen el tipo de datos longitudinales requeridos para el estudio de trayectorias laborales. ${ }^{8}$ Por estas razones se presentan aquí datos de un proyecto de investigación titulado "Trayectorias de empleo y conceptos del trabajo: trabajadores asalariados y por cuenta propia en Puebla, México", realizado entre 1990 y 1995 con la ayuda de la Asociación Alemana de Investigaciones Científicas (DFG).$^{9}$ La muestra obtenida por esta investigación es representativa en lo concerniente a las variables de "estructura por edad" y de "nivel de escolaridad" de la población ocupada en Puebla, pero respecto a las demás variables no se calculó la representatividad estadística en términos estrictos. Esto se debe, entre otros factores, al hecho de que sobre algunos datos básicos no hay conocimientos del universo total de que se trata. Por ejemplo, no había datos confiables sobre la composición numérica total del llamado sector informal urbano, y menos aún de otras variables; por lo tanto no se pudo calcular con exactitud la representatividad estadística. ${ }^{10} \mathrm{~A}$ pesar de estas limitaciones, los datos poblanos permiten un análisis en la perspectiva del enfoque biografía y sociedad y de algunos supuestos de la hipótesis de la institucionalización del curso de vida.

Un planteamiento susceptible de ser comprobado empíricamente es la hipótesis de la estandarización de los límites de tiempo entre las fases de estudios y trabajo. Según el concepto de la institucionalización del curso de vida en el transcurso del proceso de modernización, las fa-

\footnotetext{
${ }^{8}$ Véase por ejemplo el interesante trabajo de Suárez López (1992; 360) basado en la Encuesta Nacional sobre Fecundidad y Salud (Enfes) que, respecto a la trayectoria laboral, cuenta con las tres preguntas: ¿trabajó usted antes de su primer matrimonio o unión?, ¿trabajó usted después de su primer matrimonio o unión, y antes del nacimiento de su primer hijo? y ¿trabaja usted actualmente?

${ }^{9}$ Un total de 477 personas aleatoriamente seleccionadas fueron encuestadas para consignar toda su historia laboral y de vida. La mitad de los encuestados trabajó como asalariados (obreros textiles y de la rama automotriz) en el llamado sector formal, y la otra mitad trabajó por cuenta propia (talleres mecánicos, locatarios en mercados, vendedores ambulantes, en fondas o taquerías y en tiendas de abarrotes) en condiciones que los adscriben al llamado sector informal urbano -concepto que se proponía poner a prueba en esta investigación. Para más detalles véase Pries, 1992.

${ }^{10}$ Para conocer más detalles, véase Pries, 1995, capítulo Ill.1.e. El objetivo de la investigación fue captar toda la trayectoria de vida, principalmente la laboral, de ahí que las encuestas nacionales (como la Encuesta Nacional de Empleo Urbano, la Encuesta Nacional de Ingresos y Gastos de Hogares o la Encuesta Nacional de Empleo) no sean útiles porque carecen de la información correspondiente. Por varias razones no buscábamos representatividad estadística. El estudio de Puebla fue más bien una investigación explorativa.
} 
ses de estudios-educación y de actividad económica-empleo son cada vez más diferenciadas y marcadas. ¿Aprueban o reprueban este supuesto los datos de la investigación realizada en Puebla? Para poder responder a esta pregunta medimos las diferencias entre la salida del último centro de estudios y el principio del primer trabajo. La gráfica 2 muestra las frecuencias logaritmizadas con las que ocurren los diferentes valores de la variable "tiempo diferencial entre estudios y trabajo". Revela que para el universo de la muestra no tiene mucho sentido hablar de dos fases distintas y arcadas de estudios y de trabajo, porque gran parte de los encuestados ya tenía un empleo de al menos medio tiempo durante un lapso promedio de uno a dos años, pero por lo regular contaba ya con un empleo de tiempo completo cuando todavía estaba estudiando. En la gráfica los valores negativos representan estos tiempos de traslape, donde estudios y empleo se superponen, mientras que los valores positivos representan "huecos de tiempo" entre estudios y trabajo. ${ }^{11}$

Si comparamos la cohorte de edades menores (nacidos entre 1955 y 1965) con la de edades mayores (nacidos entre 1945 y 1955), tenemos que rechazar la hipótesis de una estandarización de los tiempos de paso entre el sistema educativo y el sistema productivo. Los en-

\section{GRÁFICA 2}

Tiempo entre estudios y trabajo en años (tiempo en años, frecuencias logaritmizadas)

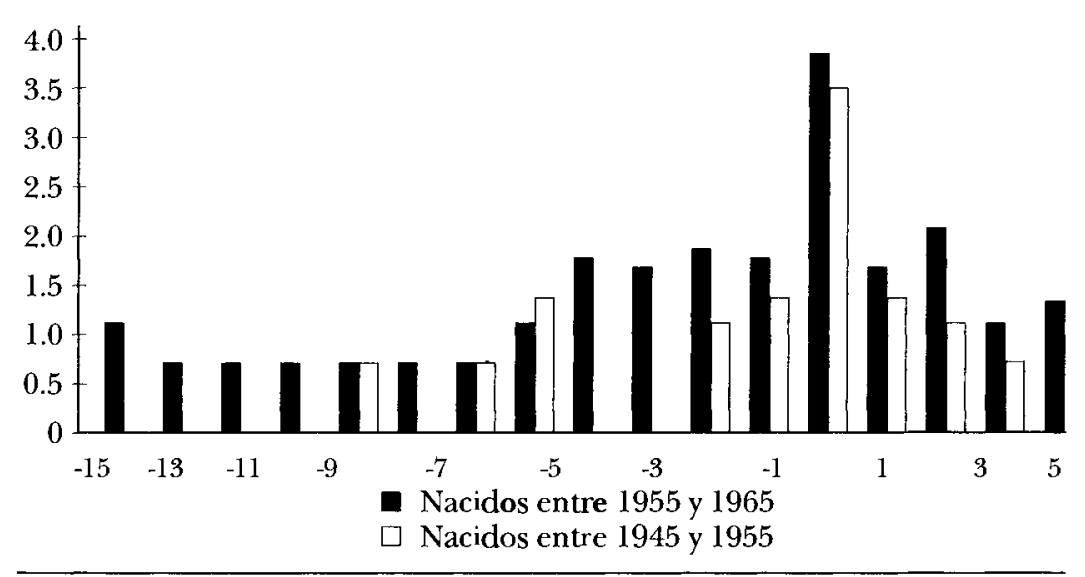

${ }^{11}$ Consideramos aquí solamente a los varones porque al incluir a las mujeres se amplía mucho el número de casos de "huecos" entre estudios y trabajo debido al hecho de que muchas mujeres encuestadas se involucraron tarde a una actividad económica. 
cuestados de menor edad presentan con mayor frecuencia amplios periodos durante los cuales los estudios y el empleo se traslapan. En el cuadro 1 se muestran algunos datos utilizando los valores absolu tos de la gráfìca 2.

Podemos notar que la edad promedio de salida de la escuela, entre las dos cohortes de edades aumentó de 15.98 a 16.43 años, al mismo tiempo que aumentó de 23 a 23.45 años el valor límite de los 5\% máximos, es decir, $95 \%$ de todos los encuestados salió de los estudios

CUADRO 1

Relación temporal entre estudios y trabajo según cohortes de edades (solamente varones, $\mathbf{n}=\mathbf{1 7 4}$ )

\begin{tabular}{llrrr}
\hline Cohorte & & $\begin{array}{r}\text { Salida } \\
\text { escuela }\end{array}$ & $\begin{array}{c}\text { Entrada } \\
\text { trabajo }\end{array}$ & $\begin{array}{r}\text { Tiempo } \\
\text { de solapo }\end{array}$ \\
\hline Nacidos & Media & 15.98 & 14.77 & 1.09 \\
entre & Desv. est. & 4.03 & 4.26 & 3.74 \\
1945 y & 5\% min. & 11.25 & 9.00 & 8.00 \\
1955 & 5\% max. & 23.00 & 22.00 & -2.75 \\
& & & & \\
Nacidos & Media & 16.43 & 14.58 & 1.79 \\
entre & Desv. est. & 3.98 & 3.82 & 4.43 \\
1955 y & 5\% min. & 11.00 & 8.00 & 12.90 \\
1965 & 5\% max. & 23.45 & 20.45 & 4.00 \\
\hline
\end{tabular}

Media: El valor promedio de este grupo.

Desv. est.: La desviación estándar mide la homogeneidad de la distribución de los valores correspondientes.

$5 \%$ min.: Indica el valor bajo el cual se quedan solamente $5 \%$ de los casos.

$5 \%$ max: Indica cuál valor está sobrepasado solamente por $5 \%$ de los casos.

Un valor negativo en "tiempo solapa" significa un "hueco" entre estudios y trabajo.

Fuente: gráfica 2.

hasta cumplir 23.45 años. Es interesante apuntar que al mismo tiempo que se alarga el tiempo promedio de estudios, se mantiene -e incluso baja un poco (de 14.77 a 14.58 años)- la edad de la primera entrada a un empleo de tiempo completo. La desviación estándar no se reduce considerablemente entre las dos cohortes (poco en la entrada al empleo, casi nula en la salida de los estudios e incluso aumenta en el tiempo de superposión entre estudios y trabajo, de 3.74 a 4.43 años). 
Con base en estos datos no se puede afirmar la hipótesis de una estandarización de los tiempos límites entre estudios y trabajo. Se alarga el periodo promedio de estudios y, al mismo tiempo, baja la edad promedio de ingreso a un trabajo. Mientras que podemos interpretar lo primero como una expresión de la tendencia generalizada a adquirir mayores niveles de estudios formales en México (como en casi todos los países del mundo), el hecho de que no aumente de manera correspondiente la edad promedio de entrada al mundo del trabajo, puede obedecer a una particularidad de México o de los países menos industrializados en general: posiblemente la crisis económica y el deterioro de los salarios reales en las últimas dos décadas obligaron a los hijos de familia a involucrarse en la fuerza de trabajo, aun cuando estuvieran todavía estudiando. ${ }^{12}$

En resumen, los datos de la investigación poblana sobre trayectorias laborales y conceptos de trabajo de trabajadores asalariados y por cuenta propia no indican un cambio claro hacia una pauta de estandarización del curso de vida. Respecto a la supuesta diferenciación más clara entre la etapa de formación-estudios y la del trabajo, no hay indicios evidentes de que ocurra este proceso en el México actual. Cabe reafirmar que los datos procesados no se refieren a un gran número de casos; son unos cuantos los que permiten ilustrar cómo se puede trabajar empíricamente sobre las hipótesis de la institucionalización del curso de vida.

\section{Consideraciones finales}

El objetivo de este artículo ha sido presentar el enfoque biografía y sociedad como un concepto integrativo e interdisciplinario para analizar estructuras y regularidades sociales en una perspectiva dinámica de cortes longitudinales. Con el ejemplo de la hipótesis de la institucionalización del curso de vida que se desarrolló y debatió en Alemania en las últimas dos décadas se mostró que desde este enfoque se pueden desarrollar diagnósticos muy interesantes y estimulantes. Por último, a pesar de contar con una muy limitada base de datos, pudimos constatar que es posible y bastante fructífero tomar este tipo de hipótesis como punto de partida para realizar investigaciones comparativas aplicadas

${ }^{12}$ Esta interpretación coincide con los hallazgos generales de Cortés y Rubalcava (1991), basados en un análisis de las Encuestas Nacionales de Ingresos y Gastos de Hogares (ENIGH) en México. 
al caso de México. Se pueden instrumentar adecuadamente dentro del marco del enfoque biografía y sociedad. Regresando a las pregun tas centrales, de entrada habremos de retomar la cuestión: ¿cómo el enfoque biografia y sociedad ayuda a conceptuar temas centrales de las ciencias sociales como son "estructura y acción", "orden y cambio social"?

Primero, el enfoque biografia y sociedad puede abrir una visión más completa y compleja de lo que es la estructuración de la sociedad. De acuerdo con esto, por estructura no entendemos casillas prefabricadas o "carriles de una vía" (o mejor dicho: de vida) de los cuales no se puede salir. La "estructura social" más bien consta de reglas de procedimiento y hábitos rutinarios que preforman la acción de los hombres. El enfoque de "estructura" trata sobre las reglas más allá (antes) de las intenciones y, en este sentido, se refiere al aspecto de la reproducción de la realidad social. Pero al mismo tiempo hay que considerar que estas reglas están abiertas tanto para las interpretaciones como para las transformaciones en la manera de actuar. ${ }^{13}$ Por lo tanto, el actuar, la acción humana, por un lado, es contingente y situativa, y por otro, es consistente y estructurada. En el contexto del tema "biografía y sociedad" es importante subrayar que la acción humana tiene el doble horizonte del tiempo: del pasado (por qué actuar) y del futuro (para qué actuar) (Fischer y Kohli, 1987: 36).

Se puede obtener una perspectiva mucho más dinámica de lo que es la realidad social estudiando retrospectivamente la dialéctica del desarrollo continuo de los hombres en la configuración compleja de los demás actores y en el correspondiente régimen de normas, prácticas, rutinas, relaciones de recursos y poderes, atendiendo a aspectos centrales como familia, género, educación, empleo, migración, etcétera. En este sentido, México cuenta con una base y tradición de investigaciones muy ricas. El reto actual es doble: mejorar los marcos teóricos y orientar el trabajo empírico hacia hipótesis fuertes y estimulantes y, al mismo tiempo, desarrollar el instrumental téorico-metodológico para superar el nivel de los simples "cortes transversales" y avanzar en "dar espacio al tiempo".

\section{Bibliografía}

Adorno, Theodor W. et al. (1972), Der Positivismisstreit in der Deutschen Soziologie, Darmstadt, Neuwied.

${ }^{13}$ Véase Fischer y Kohli, 1987: 46, y respecto al tema de la estructuración de la sociedad la obra de Anthony Giddens (1984). 
Alexander, Jeffrey et al. (eds.) (1987), The Micro-Macro-Link, Berkeley-Los Ángeles-Londres, University of California Press.

Allmendinger, Jutta (1989), Career Mobility Dynamics. A Comparative Analysis of the United States, Norway, and West Germany, Berlin, Max Planck Institut für Bildungsforschung (Studien und Berichte, 49).

Balán, Jorge et al. (1974), Las historias de vida en ciencias sociales. Teoria y técnica, Buenos Aires, Nueva Visión.

-, Harley Browning y Elizabeth Jelin (1977), El hombre en una sociedad en desarrollo, México, Fondo de Cultura Económica. [En inglés: Men in a Developing Society. Geographic and Social Mobility in Monterrey, México, Austin, Institute of Latin American Studies, 1973.]

Beck, Ulrich (1986), Risikogesellschaft. Auf dem Weg in eine andere Moderne, Frankfurt, Suhrkamp. [En inglés: Risk Society. Towards a New Modernity, Londres, SAGE Publications, 1992.]

Berger, Peter A. y Stefan Hradil (eds.) (1990), Lebenslagen, Lebensläufe, Lebensstile. (Sonderband 7 der Sozialen Welt), Göttingen, Schwartz.

Bertaux, Daniel y Martin Kohli (1984), "The Life Story Approach: A Continental View", Annual Review of Sociology, vol. 10, pp. 215-237.

Bohnsack, Ralf (1991), Rekonstruktive Sozialforschung. Einführung in Methodologie und Praxis qualitativer Forschung, Opladen, Leske \& Budrich.

Bourdieu, Pierre, Jean Claude Chamboredon y Jean Claude Passeron (1990), El oficio de sociólogo, México, Siglo XXI. [Original en francés: Le metier de sociologue, 1973.]

Bronfman, Mario (1990), "Mortalidad infantil y crisis en México", Estudios Sociológicos, vol. 8, pp. 335-350.

Brose, Hanns-Georg (1990), "Berufsbiographien im Umbruch. Erwerbsverlauf und Lebensführung von Zeitarbeitnehmern", en Karl Ulrich Mayer (ed.), Lebensverläufe und sozialer Wandel. Sonderheft 31 der Kölner Zeitschrift für Soziologie und Sozialpsychologie, Opladen, Westdeutscher Verlag, pp. 179-211.

Camp, Roderic A. (1990), Los empresarios y la política en México: una visión contemporánea, México, Fondo de Cultura Económica.

Cooper, Jennifer et al. (comps.) (1989), Fuerza de trabajo femenina urbana en México, 2 vols., México, unam/Porrúa.

Cortés, Fernando y Rosa María Rubalcava (1991), Autoexplotación:forzada y equidad por empobrecimiento, México, El Colegio de México (Jornadas, 120).

Dex, Shirley (ed.) (1991), Life and Work History Analyses: Qualitative and Quantitative Developments, Londres-Nueva York, Routledge.

Elder, Glen H. y Avsholm Caspi (1990), "Persönliche Entwicklung und sozialer Wandel. Die Entstehung der Lebensverlaufsforschung", en Karl Ulrich Mayer (ed.), Lebensverläufe und sozialer Wandel. Sonderheft 31 der Kölner Zeitschrift für Soziologie und Sozialpsychologie, Opladen, Westdeutscher Verlag, pp. 22-57. 
Elias, Norbert (1969), Über den ProzeB der Zivilisation, Bern-München. [1939.]

Elster, John (1991), El cemento de la sociedad. Las paradojas del orden social, Barcelona, Gedisa. [Original en inglés: The Cement of Society, 1989.]

Escobar Latapí, Agustín (1986), Con el sudor de tu frente. Mercado de trabajo y clase obrera en Guadalajara, Guadalajara, El Colegio de Jalisco.

Fischer, Wolfram y Martin Kohli (1987), "Biographieforschung", en Wolfgang Voges (ed.), Methoden der Biographie und Lebenslaufforschung, Opladen, Leske \& Budrich, pp. 25-49.

Foucault, Michel (1977), Überwachen und Strafen, Frankfurt, Suhrkamp. [Original en francés: 1975.]

Fuchs, Werner (1984), Biographische Forschung. Eine Einfühurng in Praxis und Methoden, Opladen, Westdeutscher Verlag.

Fuchs-Heinritz, Werner (1990), "Biographische Studien zur Jugendphase", en Karl Ulrich Mayer (ed.), Lebensverläufe und sozialer Wandel. Sonderheft 31 der Kölner Zeitschrift für Soziologie und Sozialpsychologie, Opladen, Westdeutscher Verlag, pp. 58-88.

Gamboa Ojeda, Leticia (1985), Los empresarios de ayer. El grupo dominante en la industria textil de Puebla 1906-1929, Puebla, Universidad Autónoma de Puebla.

García Guzmán, Brígida y Orlandina de Oliveira (1994), Trabajo:femenino y vida familiar en México, México, El Colegio de México.

Giddens, Anthony (1984), The Constitution of Society. Outline of the Theory of Structuration, Cambridge, Polity Press.

- et al. (1990), La teoria social hoy, Madrid, Alianza Editorial. [Original en inglés: Social Theory Today, 1987.]

González de la Rocha, Mercedes (1986), Los recursos de la pobreza. Familias de bajos ingresos de Guadalajara, Guadalajara, El Colegio de Jalisco.

Hagestad, Gunhild (1990), "Social Perspectives on the Life Course", en R. Binstock y L. George (eds.), Handbook of Aging and the Social Sciences, San Diego, Academic Press, pp. 151-168.

Herz, Thomas A. (1986), Social Mobility. An ISSC Workbook in Comparative Analysis, Frankfurt-Nueva York, Campus.

Kohli, Martin (1985), "Die Institutionalisierung des Lebenslaufs. Historische Befunde und theoretische Argumente", en Soziale Welt, vol. 37, pp. 1-29.

-_ y Günther Robert (eds.) (1984), Biographie und soziale Wirklichkeit. Neue Beiträge und Forschungsperspektiven, Stuttgart, Metzler.

Lewis, Oscar (1965), Los hijos de Sánchez; autobiografia de una familia mexicana, México, Fondo de Cultura Económica.

Long, Norman y Ann Long (eds.) (1992), Battlefields of Knocwledge. The Interlocking of Theory and Practice in Social Research and Development, LondresNueva York, Routledge.

Mayer, Karl Ulrich (ed.) (1990), Lebensverläufe und sozialer Wandel. Sonderheft 31 der Kölner Zeitschrift für Soziologie und Sozialpsychologie, Opladen, Westdeutscher Verlag. 
Mummert, Gail (ed.) (1990), Población y trabajo en contextos regionales, Zamora, El Colegio de Michoacán.

Muñoz, Humberto, Orlandina de Oliveira y Claudio Stern (1977), Migración y desigualdad social en la ciudad de México, México, El Colegio de México/Instituto de Investigaciones Sociales, UNAM.

Oevermann, U., T. Allert, E. Konau y J. Krambeck (1979), "Die Methodologie einer 'objektiven' Hermeneutik» und ihre allgemeine forschungslogische Bedeutung in den Sozialwissenschaften", en H. G. Söffner (comp.), Interpretative Verfahren in den Sozial und Textwissenschaffen, Stuttgart, pp. 352-434.

Oliveira, Orlandina de, Marielle Pepin Lehalleur y Vania Salles (comps.) (1989a), Grupos domésticos y reproducción cotidiana, México, unam/Porrúa/El Colegio de México.

- (coord.) (1989b), Trabajo, poder y sexualidad, México, El Colegio de México.

Pries, Ludger (1992), Hacia una sociología del empleo (Tres ensayos, Avances de Investigación núm. S-1), Puebla, El Colegio de Puebla.

- (1993), Transformación industrial productiva en una perspectiva comparativa: $\underset{\epsilon}{ }$ modernización reflexiva, modernización truncada o modernización híbrida?, Tijuana, El Colegio de la Frontera Norte (Cuaderno de Discusión, 9).

(1995), "Wege und Visionen von Erwerbsarbeit in Mexiko. Erwerbsverläufe und Arbeitsorientierungen abhängig und selbständig Beschäftigter in einem semi-industrialisierten Land" ("Caminos y conceptos del empleo en México. Trayectorias laborales y orientaciones en el trabajo de trabajadores asalariados y por cuenta propia en un país semi-industrializado"), tesis de habilitación, Nüremberg, Universidad de Erlangen.

Pujadas Muñoz, Juan José (1992), El método biográfico: el uso de las historias de vida en ciencias sociales, Madrid, Centro de Investigaciones Sociológicas.

Riesman, David (1958), Die cinsame Masse, Reinbek, Rowohlt. [Original en inglés: 1950.]

Saltalamacchia, Homero R. (1992), La historia de vida: reflexiones a partir de una experiencia de investigación, Caguas, cyus.

Schütze, Fritz (1983), "Biographieforschung und Narratives Interview", Neue Praxis, núm. 3, pp. 283-293.

Suárez López, Leticia (1992), "Trayectorias laborales y reproductivas: una comparación entre México y España”, Estudios Demográficos y Urbanos, vol. 7, núms. 2-3 (20-21), pp. 359-375.

Thomas, W. I. y F. Znaniecki (1921), The Polish Peasant in Europe and America, 2 vols., Nueva York, Dover.

Torres B., Mariano (1994), La familia Maurer de Allixco, Puebla. Entre el porfiriato y la revolución, México, Consajo Nacional para la Cultura y las Artes (Conaculta).

Voges, Wolfgang (ed.) (1987), Methoden der Biographie und Lebenslaufforschung, Opladen, Leske \& Budrich. 
Weber, Max (1980), Wirtschaft und Gesellschaft, Tübingen, Mohr. [1922.]

Whyte, William Foote (1943), Street Corner Society. The Social Structure of an Italian Slum, Chicago, University of Chicago Press.

Wilson, Fiona (1990), De la casa al taller. Mujeres, trabajo y clase social en la industria textil y del vestido, Zamora, El Colegio de Michoacán. 
